\title{
STATISTICAL ANALYSIS OF LONG-RANGE DEPENDENT RANDOM PROCESSES AND FIELDS
}

\author{
HUDA MOHAMMED ALOMARI
}

(Received 14 September 2019; first published online 8 January 2020)

\section{Mathematics subject classification: primary 62F12; secondary 60G60, 62M30.}

Keywords and phrases: long-memory parameter, Ibragimov minimum contrast estimator, tapered data, Gegenbauer random field, wavelet transforms, filter, estimators of parameters, cyclic long memory.

The thesis deals with estimation of long-memory parameters of random processes and fields. Long-memory behaviour of data has been reported in various applications, for example in finance, internet traffic and hydrology. It is known that estimators of parameters of long-memory and especially seasonal long-memory processes have complex non-Gaussian asymptotic distributions (see [5]).

In the first part, the consistency and asymptotic normality for the so-called Ibragimov minimum contrast estimators based on tapered data are investigated for various models of Gaussian random processes and fields. Periodogram-based estimators of parameters are used. Tapering is employed to reduce the bias of the periodogram in the spatial data. It is known that tapering data reduces leakage effects, especially when spectral densities contain high peaks. The use of tapers can lead to bias reduction, which is a key issue in spatial statistics, by helping to remove the socalled 'edge effects'.

The thesis develops a new methodology to verify assumptions for consistency and asymptotic normality of long-memory parameter estimators. The cases of Gaussian fractional autoregressive processes and Gegenbauer random fields are considered. This methodology is applicable for short- and long-range dependent cases. We review some results from the literature on the Whittle estimators based on tapered data, with a particular focus on the conditions on tapers which help to control the bias of estimators. Then we study the case of the Whittle estimators with tapered data for weakly and strongly dependent random fields.

It is proved that minimum contrast estimators of the parameters are consistent estimators. Various particular cases of spectral densities and tapers are considered.

Thesis submitted to La Trobe University in December 2018; degree approved on 26 April 2019; principal supervisor Andriy Olenko, co-supervisor Luke Prendergast.

(C) 2020 Australian Mathematical Publishing Association Inc. 
It is shown that they satisfy rather general conditions required for consistency. For the case of long-memory random fields, the central limit theorem for spectral functionals based on the tapered data is obtained. These results generalise several well-known approaches in this field which are based on contrast estimators (see [4]).

The second part of the thesis develops the approaches proposed in [3, 6]. The paper [6] used wavelet transforms to estimate parameters of cyclic long-memory time series. Simulation studies were performed to validate the approach and to compare it with other techniques. Unfortunately, there were no rigorous studies to justify the method and establish statistical properties of the estimators, except for the case of a singularity at the origin. We address this problem and take the first steps in developing simultaneous estimators for seasonality and long-memory parameters. Gegenbauertype cyclic long-memory semiparametric models are discussed. The Gegenbauer-type spectral density $f(\lambda) \sim C\left|\lambda^{2}-v^{2}\right|^{-2 \alpha}$ has singular asymptotic behaviour around its poles $\pm v$ when $\lambda \rightarrow \pm v$. The thesis derives simultaneous estimators of the parameters $\lambda$ and $\alpha$.

We use the idea from [3] to develop the first estimation equation. Namely, we study asymptotic properties of a filter transformation of cyclic long-memory processes. To get the second estimation equation, we propose a new approach that is based on asymptotic behaviour of increments of the filter transformation. Finally, we investigate properties of the solutions to the estimation equations and propose adjusted statistics for both the cyclic and long-memory parameters. The methodology developed includes wavelet transformations as a particular case. Therefore, it is potentially very useful for real applications as it can employ the existing wavelet methods and software which are more powerful and faster than programs for numerical integration and optimisation required by the ordinary least-squares, maximum-likelihood estimation and multicriteria evaluation methods.

In addition to the theoretical results, the thesis develops $\mathrm{R}$ code to simulate Gegenbauer-type long-memory random fields and to study performance of the estimation techniques introduced. The thesis provides numerical studies of the convergence properties of estimators of the parameters of Gegenbauer random fields. We also develop R programs to simulate and study filtered cyclic long-range dependent processes.

Some of the results obtained in the thesis have been published in $[1,2]$.

\section{References}

[1] H. M. Alomari, A. Ayache, M. Fradon and A. Olenko, 'Estimation of seasonal long-memory parameters', Scand. J. Stat., to appear.

[2] H. M. Alomari, M. P. Frías, N. N. Leonenko, M. D. Ruiz-Medina, L. Sakhno and A. Torres, 'Asymptotic properties of parameter estimates for random fields with tapered data', Electron. J. Stat. 11(2) (2017), 3332-3367.

[3] J. M. Bardet and P. R. Bertrand, 'A non-parametric estimator of the spectral density of a continuoustime Gaussian process observed at random times', Scand. J. Stat. 37(3) (2010), 458-476.

[4] R. Espejo, N. Leonenko, A. Olenko and M. Ruiz-Medina, 'On a class of minimum contrast estimators for Gegenbauer random fields', Test 24(4) (2015), 657-680. 
[5] A. Olenko, 'Limit theorems for weighted functionals of cyclical long-memory random fields', Stoch. Anal. Appl. 31(2) (2013), 199-213.

[6] B. Whitcher, 'Wavelet-based estimation for seasonal long-memory processes', Technometrics 46(2) (2004), 225-238.

HUDA MOHAMMED ALOMARI,

Al-Baha University, Al-Baha,

Saudi Arabia

e-mail: haldrawshah@bu.edu.sa 